\section{Kaum bekannte Biologie: vom Rätsel der Hirnmetastasen}

$\mathrm{H}$ irnmetastasen sind ein spätes Ereignis im Krankheitsverlauf, unter anderem beim Mammakarzinom. Doch der Metastasierungsprozess mit seiner klonalen Entwicklung ist keineswegs wie lange angenommen linear, berichtete Prof. Tobias Pukrop, interdisziplinäre Onkologische Tagesklinik, Universitätsklinikum Regensburg. Bei verschiedenen Tumorentitäten konnte inzwischen gezeigt werden, dass sich die klonale Entwicklung früh verästelt. Die Folge: Hirnmetastasen haben ein ganz anderes genetisches Profil als der Primarius und der Stammzellklon ist meist nicht mehr nachweisbar. Die manifeste Hirnmetastase sei ferner das Ende einer langen Entwicklung. Möglicherweise haben es schon bei Diagnosestellung einzelne Tumorzellen geschafft, die Blut-HirnSchranke und endotheliale Abwehrmechanismen des Gehirns zu überwinden, so Pukrop. Die Ansiedlung hat also möglicherweise längst stattgefunden, das Gehirn ist bereits kolonisiert, auch wenn die Makrometastasierung erst viel später auftritt. Die Therapie des Primarius beeinflusst diese weitere Entwicklung nicht. Wahrscheinlich führen die anderen $\mathrm{Ab}$ wehrmechanismen in verschiedenen Organen auch zu einer spezifischen Selektion von Klonen. Pukrop sprach von einer maximalen Heterogenität - nicht nur Primarius und Hirnmetastasen haben wenig gemein, auch eine Organmetastase habe ihr eigenes Mutationsmuster. Das könnte auch der Grund sein, warum viele zielgerichtete Therapien zwar das progressionsfreie, nicht aber das Gesamtüberleben verlängern. Die Mutationsbestimmung des Primarius alleine scheint oft ungenügend, zumindest progrediente Metastasen müssen künftig vielleicht häufiger biopsiert oder reseziert werden.
Über die Biologie von Hirnmetastasen sei derzeit noch viel zu wenig bekannt.

Zumindest für ZNS-Metastasen beim Mammakarzinom ist Prof. Volkmar Müller, Universitätsklinikum Hamburg Eppendorf, dennoch optimistisch. Verschiedene, meist HER2-gerichtete Tyrosinkinaseinhibitoren, ein trifunktionaler Antikörper sowie ein Vakzin befinden sich in früher klinischer Prüfung. Da Hirnmetastasen von verschiedenen Primärtumoren unterschiedlich sensibel sind, sollten Studien organspezifisch angelegt werden. Müller lud zur deutschen Registerstudie BMBC zu ZNS-Metastasen beim Mammakarzinom ein. Im Rahmen der Studie sollen prospektiv und retrospektiv Daten zu Erkrankungsverläufen von Patientinnen mit Hirnmetastasen eines Mammakarzinoms erhoben werden. Die Daten sollen helfen, die Ursachen für die Entstehung von Hirnmetastasen bei Brustkrebs besser zu verstehen. Friederike Klein

"State of the Art: Hirnmetastasen und spinale Metastasen", 25.2.2016

\section{Krebs im hohen Alter: Scores für eine gezielte Therapie}

D ie gesundheitliche Gesamtsituation älterer Menschen werde in der Krebstherapie oft nicht genug berücksichtigt, sagte Prof. Yon-Dschun Ko, JohanniterKrankenhaus Evangelische Kliniken Bonn. $40 \%$ der 70-Jährigen sterben innerhalb von zehn Jahren an nicht onkologischen Erkrankungen. Die Wirksamkeit einer adjuvanten Therapie, durch die 2-4\% mehr Patienten zehn Jahre lang leben, werde allein deswegen schon statistisch ausgedünnt. Infolge eingeschränkter Leber-, Nieren- und Hirnfunktion treten im Alter unerwünschte Wirkungen auch rascher ein und sind schwerer rückgängig zu machen. „Wir müssen uns deswegen bei alten Krebspatienten immer fragen, ob eine Therapie eigentlich noch relevant ist", so Ko. Entscheidend sei, die nicht krebsbezogene Gesamtprognose zu bewerten. Doch wie können diejenigen erkannt werden, die noch von einer Maximaltherapie profitieren? Imke Ortland, Klinische Pharmazie, Universität Bonn, plädierte für den Einsatz onkogeriatrischer Scores wie den CRASH- oder CARG-Score, die krebs- und krebstherapiebezogene sowie altersbezogene Faktoren einbeziehen und in Bonn derzeit klinisch evaluiert werden. In der Pilotphase mit 20 Krebspatienten jenseits der 70 bescheinigten beide Scores jeweils drei von vier Patienten ein mittleres bis hohes Risiko für starke unerwünschte Wirkungen. Die behandelnden Ärzte dagegen hielten 16 von 20 Patienten fit genug für eine Maximaltherapie. Bei über 100 Patienten wird jetzt prospektiv überprüft, ob die Scores oder Ärzte die Patienten besser einschätzen. Prof. Dr. Ulrich Jaehde, Pharmazeutisches Institut der Universität Bonn, stellte eine Studie vor, in der bei Krebspatienten in Abhängigkeit vom Risiko für unerwünschte Wirkungen wie Mucositis, Nausea/Emesis, Schmerzen oder Fatigue bestimmte Medikationsmanagementmodule durchlaufen wurden. Auftretende Probleme sollten früh er- kannt werden. Unabhängig vom Alter der Patienten ließ sich dadurch das Auftreten schwerer unerwünschter Wirkungen im Mittel um einen Therapiezyklus verzögern, so Jaehde. Im nächsten Schritt soll das Bonner Medikationsmanagement jetzt um onkogeriatrische Assessments erweitert werden. Philipp Grätzel von Grätz

"State of the Art: Medikationsmanagement beim älteren Krebspatienten", 26.2.2016

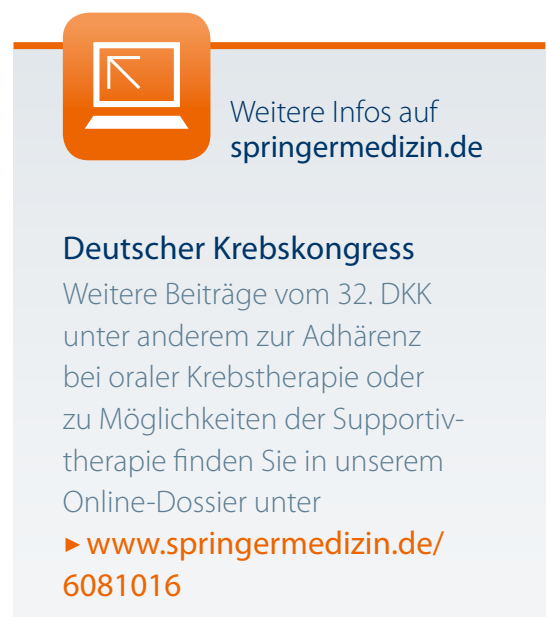

